\title{
Beam conditioning for free electron lasers: Consequences and methods
}

\author{
A. Wolski, G. Penn, A. Sessler, and J. Wurtele* \\ Ernest Orlando Lawrence Berkeley National Laboratory, Berkeley, California 94720, USA
}

(Received 17 October 2003; published 3 August 2004)

\begin{abstract}
The consequences of beam conditioning in four example cases [VISA, a soft x-ray free-electron laser (FEL), LCLS, and a "Greenfield" FEL] are examined. It is shown that in emittance limited cases, proper conditioning reduces sensitivity to the transverse emittance and, furthermore, allows for stronger focusing in the undulator. Simulations show higher saturation power, with gain lengths reduced by a factor of 2 or more. The beam dynamics in a general conditioning system are studied, with "matching conditions" derived for achieving conditioning without growth in the effective emittance. Various conditioning lattices are considered, and expressions derived for the amount of conditioning provided in each case when the matching conditions are satisfied. These results show that there is no fundamental obstacle to producing beam conditioning, and that the problem can be reduced to one of proper lattice design. Nevertheless, beam conditioning will not be easy to implement in practice.
\end{abstract}

DOI: 10.1103/PhysRevSTAB.7.080701

PACS numbers: 41.60.Cr, 29.27.-a

\section{INTRODUCTION}

Successful operation of proposed short-wavelength free-electron lasers (FELs) demands electron beams with very small transverse emittance. Particles with large betatron amplitude will tend to slip back with respect to a nominal particle with zero betatron amplitude, and thus can fall out of phase with the radiation produced in an undulator, limiting the gain of an FEL. It has been known for some time that it is possible in principle to ease the requirements on the transverse emittance by introducing a correlation between particle energy and betatron amplitude [1]. Increasing the energy, and hence the longitudinal velocity, of particles with large betatron amplitudes compensates for the phase slip resulting from the betatron amplitude. Thus, an appropriate correlation between betatron amplitude and particle energy in the undulator can ensure that the necessary phase relationship between particles in the beam and the radiation is maintained, allowing FEL operation with larger electron beam emittances than would otherwise be possible.

The analysis in Ref. [1] was limited to a bunch consisting of particles all having the same longitudinal coordinate at the conditioner. Recent work [2] suggested that any attempt to "condition" a nonzero length beam for an FEL by introducing the correct correlation between betatron amplitude and energy inevitably results in betatron mismatches at different longitudinal positions along the bunching, causing an increase in the effective transverse emittance. In parameter regimes of interest for proposed facilities, this effective emittance increase was found to be sufficiently large as to outweigh the beneficial effect of conditioning, and even to prevent any operation of the FEL. Here, we show that the increase in the

\footnotetext{
*Also at Department of Physics, University of California, Berkeley, CA.
}

effective emittance is not, in fact, inevitable, and we present designs of systems where the required conditioning is achieved without increase in the effective emittance. Simulation results for the most promising method are the same as that for an ideally conditioned beam.

In Sec. II we consider the potential benefits of beam conditioning in the context of proposed and possible facilities, and suggest some interesting parameter regimes. We then proceed in Sec. III to consider the source of the effective emittance growth found by the authors of Ref. [2], and to show the strategy for avoiding this problem. In Sec. IV we give examples of various systems that may be used to achieve conditioning. In Sec. V we simulate the output from one of the more promising lattice designs and show that a real system is as effective as the ideal beam conditioning assumed in Sec. II. In this paper we focus on using conventional elements and lattice configurations to achieve beam conditioning; it is also possible to use "exotic" laser or laser/plasma methods as described in Ref. [3].

\section{CONSEQUENCES OF BEAM CONDITIONING FOR FEL PERFORMANCE}

Optimal FEL output radiation from an electron beam is achieved when the electrons have a specific average forward velocity; after each undulator period, electrons should fall behind the laser field by one wavelength. This implies a resonance condition which is often expressed as a resonant energy, assuming zero transverse betatron amplitude. However, for a beam that has nonzero emittance, there is a spread in forward velocity associated with the path-length differences from betatron motion. This results in a spread in forward velocities which, when too large, limits FEL performance. For FELs that are emittance limited, more electrons can be brought into resonance, with the correct forward velocity, by introduc- 
ing a correlation between transverse amplitude and energy. This correlation improves performance while leaving the intrinsic energy spread and emittance of the electron beam unchanged.

For a particle having zero transverse amplitude, the average angle inside of an undulator is $a_{U} / \gamma$, where $m_{e} c^{2} \gamma$ is the electron energy, $m_{e}$ is the electron mass, $c$ is the speed of light, and $a_{U}=e B_{\mathrm{rms}} \lambda_{U} / 2 \pi m_{e} c$ is the normalized strength of the undulator; here, $B_{\mathrm{rms}}$ is the rms value of the undulator field on axis, and $\lambda_{U}$ is the period of the undulator. In the limit of large $\gamma$, the angle is roughly the same as $v_{\perp} / c$, where $\vec{v}$ is the velocity. Hence, taking the average over an undulator period,

$$
\frac{\overline{\boldsymbol{v}}_{z}^{2}}{c^{2}}=1-\frac{1}{\gamma^{2}}-\frac{\bar{v}_{\perp}^{2}}{c^{2}}=1-\frac{1+a_{U}^{2}}{\gamma^{2}} .
$$

The slippage after one undulator period, $\lambda_{U}$, is given by $\left(1-\bar{v}_{z} / c\right) \lambda_{U}$. To be in resonance, this should be equal to the desired laser wavelength $\lambda$. To lowest order in $1 / \gamma^{2}$,

$$
\lambda=\left(1-\frac{\bar{v}_{z}}{c}\right) \lambda_{U} \simeq \frac{1+a_{U}^{2}}{2 \gamma^{2}} \lambda_{U} .
$$

An electron at nonzero amplitude will have an additional angle due to its betatron motion. This angle will add in quadrature to the angle in Eq. (1). When the variation of the undulator field with radius is taken into account, this leads to the condition

$$
\lambda=\frac{1}{2}\left(\frac{1+a_{U}^{2}}{\gamma^{2}}+\frac{2 J_{x}}{\beta_{x}}+\frac{2 J_{y}}{\beta_{y}}\right) \lambda_{U},
$$

where $J_{x}, J_{y}$, are the transverse actions. In terms of the Twiss parameters,

$$
J_{x}=\frac{1}{2}\left[\frac{x^{2}}{\beta_{x}}+\beta_{x}\left(\frac{p_{x}}{P_{0}}+\frac{\alpha_{x} x}{\beta_{x}}\right)^{2}\right],
$$

where $P_{0}$ is the reference momentum, $p_{x}$ is the canonical transverse momentum, and similarly for $J_{y}$. The average value of $J_{x}$ in a beam is equivalent to the geometric emittance $\epsilon_{x}$; the normalized emittance $\epsilon_{N}=\gamma \epsilon_{x}$. Below we assume negligible $\alpha_{x}$ and $\alpha_{y}$, and that $\beta_{x}=\beta_{y}=\beta$.

FEL performance is limited by the spread in resonant wavelength when either the relative energy spread, $\sigma_{\gamma} / \gamma$, or emittance-related bandwidth, $\epsilon_{N} \lambda_{U} / 4 \beta \gamma \lambda$, is comparable to the "FEL parameter" [4], which reflects the tolerance of an FEL to deviations from resonance. When transverse emittance is the limiting factor, more particles can be brought into resonance by introducing a correlation between transverse amplitude and energy,

$$
\frac{\Delta \gamma}{\gamma}=\kappa_{x} J_{x}+\kappa_{y} J_{y}
$$

The spread in the right-hand side of Eq. (2) across the particle distribution is minimized if this correlation is chosen such that $\kappa_{x}=\kappa_{y}=\kappa$, with

$$
\kappa=\frac{1}{\beta} \frac{\gamma^{2}}{1+a_{U}^{2}}=\frac{1}{2 \beta} \frac{\lambda_{U}}{\lambda} .
$$

Values of $\kappa$ required for proper conditioning are typically of the order of $10-100 \mu \mathrm{m}^{-1}$. A value of $\kappa=1 \mu \mathrm{m}^{-1}$ implies that for a beam with $1 \mu \mathrm{m}$ normalized emittance in both transverse planes, a typical electron must have just over $1 \mathrm{MeV}$ more energy than a zero amplitude particle in order to be resonant at the same wavelength.

When the beam is more strongly focused, so that $\beta \ll \gamma \lambda_{U} / \pi a_{U}$, the variation of magnetic field with position in the bunch is not important. However, when the gain length is shorter than a betatron period, instead of averaging over angle the optimal conditioning is to adjust particles to be resonant at their peak angles, because this occurs when they are close to the axis, which is also where the laser field is strongest. This leads to the same conditioning parameter as above.

Below we consider several examples of FEL parameters to see how conditioned beams perform differently from unconditioned beams. In all cases, the two transverse directions have identical beta functions and we take the conditioning parameter $\kappa_{x}=\kappa_{y}=\kappa$. The expression for conditioning given above has been verified by simulations to be the optimal value. Note that reducing the beta function of the beam in an FEL requires stronger conditioning for proper matching. Simulations for FEL amplifier performance were performed using the GENESIS code [5].

In the following figures, red is used for the nominal emittance, green for twice the nominal emittance, and black for higher emittances. Points represent unconditioned beams and lines represent conditioned beams. Because conditioned beams are optimized at smaller beta functions, results using the best value for the beta function for a conditioned beam are represented as lines with "+" symbols. Conditioned beams are taken to have the optimal conditioning parameter in each case.

We consider four examples: VISA [6], a soft x-ray FEL [7], LCLS [8], and a "Greenfield" FEL [9]. Table I gives the parameters used in the simulation in each case.

\section{A. VISA experiment}

Results are shown in Fig. 1 for various emittances at or above the nominal value, for both conditioned and unconditioned beams. The optimum gain length is roughly $16 \mathrm{~cm}$. It is seen that at nominal values the performance is not limited by emittance, and conditioning has little effect until the emittance is increased by a factor of 4 .

\section{B. Soft x-ray FEL}

Figure 2 shows results for nominal and twice nominal emittance, for both conditioned and unconditioned 
TABLE I. Parameters used in modeling four FELs.

\begin{tabular}{|c|c|c|c|c|c|}
\hline & VISA & $\begin{array}{l}\text { Soft } \\
x \text {-ray }\end{array}$ & LCLS & $\begin{array}{l}\text { Greenfield } \\
\text { low energy }\end{array}$ & $\begin{array}{l}\text { Greenfield } \\
\text { high energy }\end{array}$ \\
\hline Radiation wavelength [nm] & 840 & 1 & 0.15 & 0.04 & 0.04 \\
\hline Electron beam energy [GeV] & 0.070 & 2.5 & 14.3 & 12.1 & 27.8 \\
\hline Fractional energy spread $\left[10^{-4}\right]$ & 8 & 4 & 1 & 1.2 & 1 \\
\hline Normalized emittance $[\mu \mathrm{m}]$ & 2.1 & 2 & 1.2 & 0.1 & 0.1 \\
\hline Peak current $[\mathrm{kA}]$ & 0.24 & 0.5 & 3.4 & 3.5 & 3.5 \\
\hline Undulator period $[\mathrm{cm}]$ & 1.8 & 2.5 & 3 & 3 & 3 \\
\hline Undulator parameter $a_{U}$ & 0.89 & 0.96 & 2.62 & 0.71 & 2.62 \\
\hline Beta function $[\mathrm{m}]$ & 0.29 & 4.8 & 17.5 & 17.5 & 17.5 \\
\hline Conditioning parameter $\left[\mu \mathrm{m}^{-1}\right]$ & 0.036 & 2.6 & 5.8 & 22 & 22 \\
\hline
\end{tabular}

beams, in a soft $\mathrm{x}$-ray FEL. The optimum gain length is roughly $7 \mathrm{~m}$, while for an unconditioned beam the gain length is twice as long. There is some degradation of the FEL performance at higher emittance for the conditioned beam, but the performance is still much improved over the nominal, unconditioned case.

\section{LCLS project}

Figure 3 shows the variation of FEL performance with beta function; the unconditioned beam does not improve as the focusing is made stronger, but the conditioned beam has a shorter gain length and higher saturated power for beta functions down to $4.4 \mathrm{~m}$. The optimum gain length is $2.5 \mathrm{~m}$, while for the unconditioned beam the gain length is $5 \mathrm{~m}$.

Figure 4 shows the variation of LCLS performance with emittance; the unconditioned beam performs far worse at higher emittances, while even at 4 times nominal emittance the conditioned beam performs as well as the

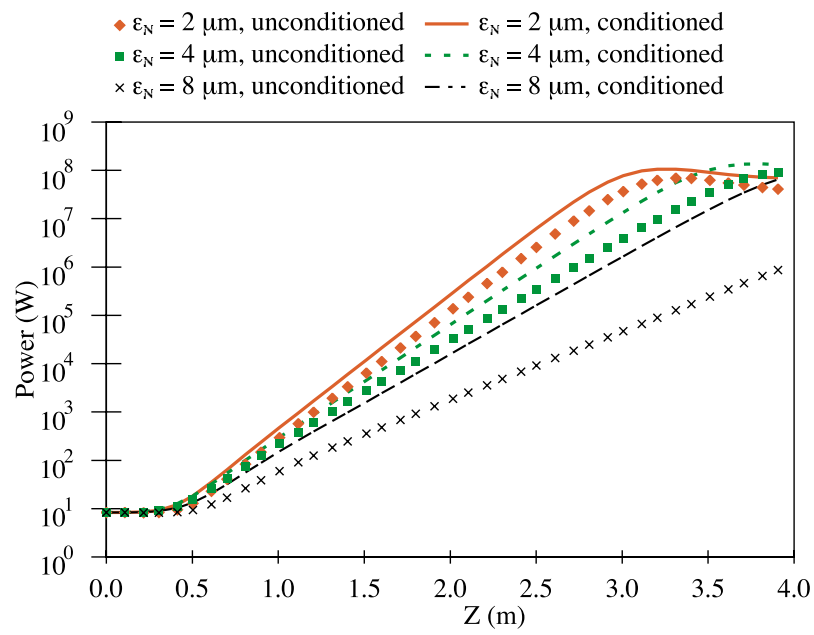

FIG. 1. (Color) Radiation power as a function of undulator length in the VISA FEL. Conditioned and unconditioned beams at different emittances are compared.

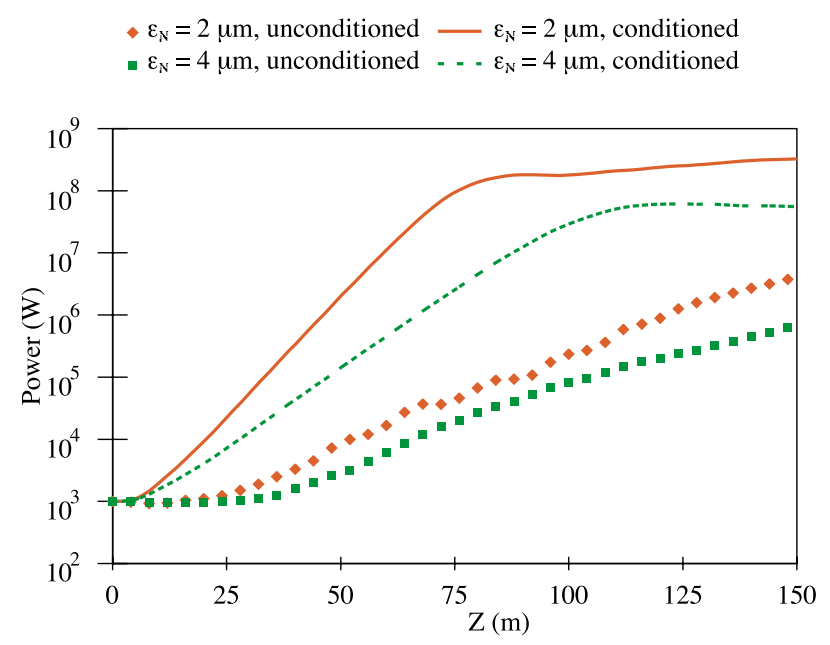

FIG. 2. (Color) Radiation power as a function of undulator length in the soft $\mathrm{x}$-ray FEL. Conditioned and unconditioned beams at different emittances are compared.

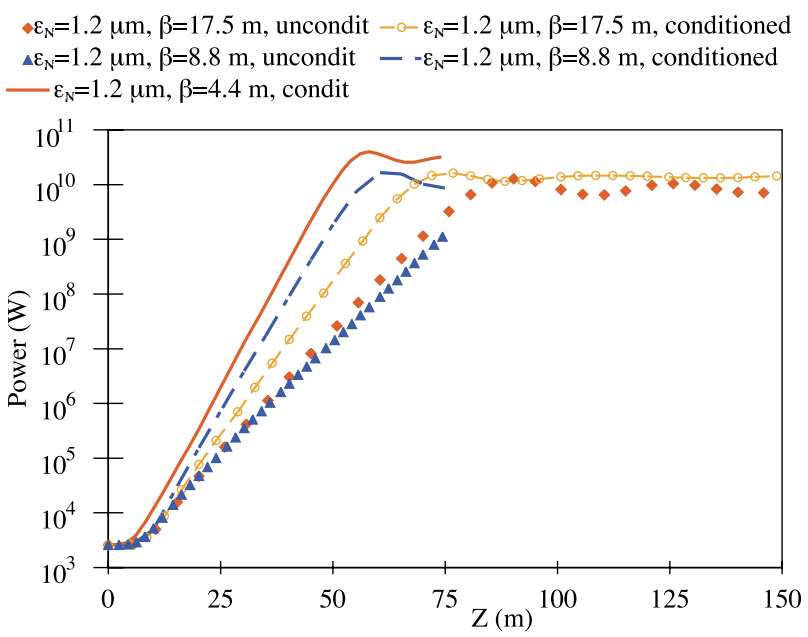

FIG. 3. (Color) Radiation power as a function of undulator length for LCLS, with different beta functions, and with conditioned and unconditioned beams. 


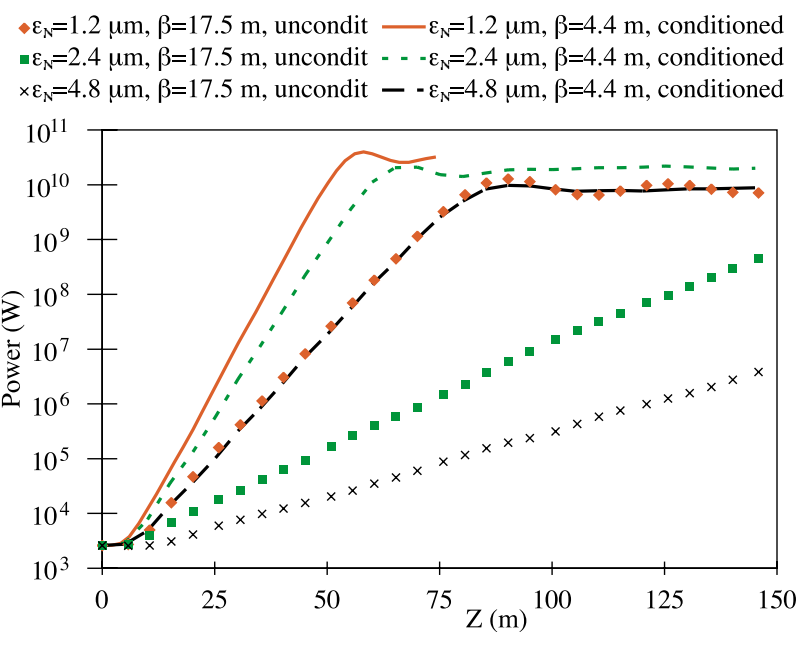

FIG. 4. (Color) Radiation power as a function of undulator length for LCLS, with different values of beam emittance, beta function, and for conditioned and unconditioned beams. Note that a conditioned beam with 4 times larger emittance (black line) achieves the same performance as the nominal case (red diamonds).

unconditioned beam at nominal emittance. Note that at smaller beta functions, the conditioning parameter is proportionately larger, with a value of $23.2 \mu \mathrm{m}^{-1}$ for a $4.4 \mathrm{~m}$ beta function.

\section{Greenfield FEL}

Future FELs may be imagined that reach wavelengths of $0.1 \AA$. Using conventional design criteria this would require ultrasmall emittances of the order of $0.1 \mu \mathrm{m}$. Conditioning allows us to reach close to Greenfield FEL design performance using a beam with an order of magnitude larger emittance and smaller beta functions.

We consider two parameter sets for Greenfield FELs (see Table I). In both cases, FEL performance for conditioned beams improves as the beta function is reduced from the nominal design value of 17.5 to $4.4 \mathrm{~m}$. For the smaller beta function the conditioning parameter is $88 \mu \mathrm{m}^{-1}$. The results are shown in Figs. 5 and 6. At $12 \mathrm{GeV}$, for a normalized emittance of $0.1 \mu \mathrm{m}$, the gain length is $3.2 \mathrm{~m}$. At emittances of $1.2 \mu \mathrm{m}$ a combination of beam conditioning and stronger focusing yields a gain length of $6 \mathrm{~m}$, with a lower saturation level. At $28 \mathrm{GeV}$, for a normalized emittance of $0.1 \mu \mathrm{m}$, the gain length is $3 \mathrm{~m}$. At emittances of $1.2 \mu \mathrm{m}$, a combination of beam conditioning and stronger focusing yields a gain length of $5 \mathrm{~m}$, with a slightly lower saturation level.

Beam conditioning requires large nonlinear correlations to be introduced into the electron distribution in order to minimize the number of particles with large deviations from the resonance condition for high emittance beams. Proper conditioning then reduces sensitivity to beam emittance and allows stronger focusing in the undulator. Simulations show gain lengths a factor of 2

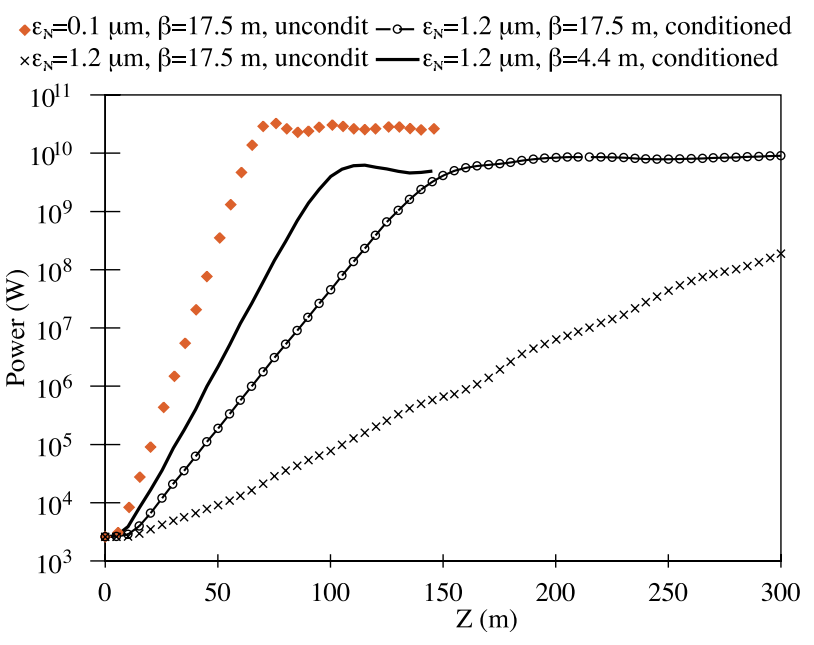

FIG. 5. (Color) Radiation power as a function of undulator length for a "Greenfield" FEL at $12 \mathrm{GeV}$. Different beta functions and emittances are shown, with conditioned and unconditioned beams.

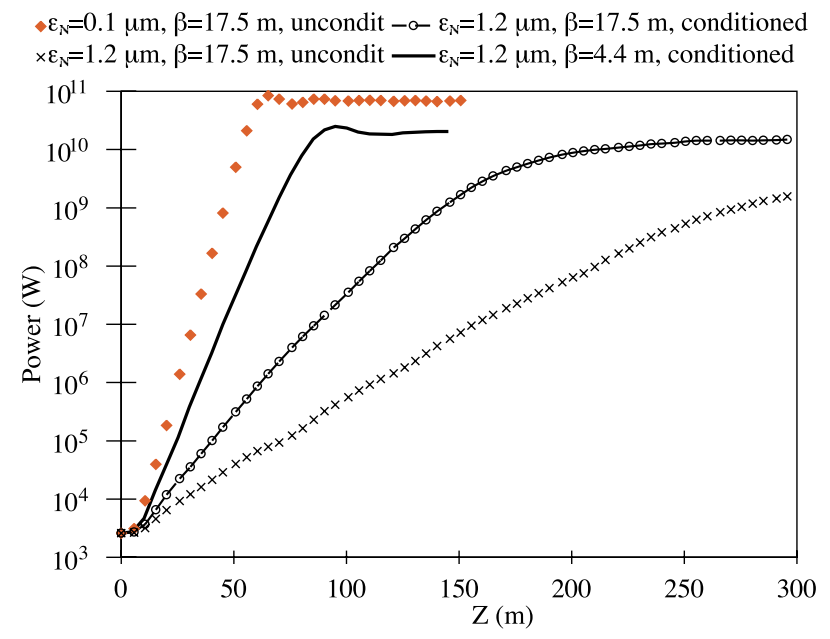

FIG. 6. (Color) Radiation power as a function of undulator length for a Greenfield FEL at $28 \mathrm{GeV}$. Different beta functions and emittances are shown, with conditioned and unconditioned beams.

shorter, with higher saturated power, compared to unconditioned beams. The benefits of beam conditioning are seen to be applicable to a wide range of FEL designs.

\section{BEAM DYNAMICS IN CONDITIONING SYSTEMS}

We can show in a straightforward fashion that conditioning may be achieved in a symplectic system without increase in the effective transverse emittance of the beam. For simplicity, we consider particle motion only in the longitudinal and one transverse plane; it will be seen that the extension to two transverse planes is trivial. We use canonical action-angle variables $(J, \phi)$ in the transverse 
plane, and canonical variables $(z, \delta)$ in the longitudinal plane, with distance $s$ along a reference trajectory as the independent variable. The reference trajectory is the path of a particle with $J=\delta=0, z$ is the displacement along the reference trajectory of the selected particle with respect to a particle following the reference trajectory, and $\delta$ is the energy deviation of the selected particle with respect to a particle following the reference trajectory.

Let us consider a system of length $L$ defined by the integrable Hamiltonian:

$$
H=\frac{\mu}{L} J+2 \pi z \frac{\tilde{\xi}}{L} J .
$$

The transformation of phase space variables following from this Hamiltonian is

$$
\begin{aligned}
J_{L} & =J_{0}, \quad z_{L}=z_{0}, \quad \phi_{L}=\phi_{0}+2 \pi \tilde{\xi} z_{0}, \\
\delta_{L} & =\delta_{0}+2 \pi \tilde{\xi} J_{0} .
\end{aligned}
$$

The subscript 0 denotes values at the beginning of the conditioning system, and the subscript $L$ denotes values at the end. Since the angle and energy deviation variables do not appear explicitly in the Hamiltonian, the action and time variables are conserved. We note that the energy deviation increases by an amount proportional to the action, so a beam of particles where the energy deviation and action are initially uncorrelated acquires a correlation between these variables:

$$
\left\langle\delta_{L} J_{L}\right\rangle=2 \pi \tilde{\xi}\left\langle J_{0}^{2}\right\rangle .
$$

Thus the above Hamiltonian gives the required conditioning.

We now need to consider what has been the effect on the matching conditions and the emittance of the bunch. In general, the Twiss parameters are functions of the energy deviation of the particle, but it is possible to design a beam line where the energy dependence vanishes (at least to first order) at some point in the lattice. This is discussed further in Sec. IVA. In this case, the beam moments are correctly given by

$$
\begin{aligned}
\left\langle x^{2}\right\rangle & =\beta \epsilon, \quad\left\langle x p_{x}\right\rangle=-\alpha \epsilon P_{0}, \\
\left\langle p_{x}^{2}\right\rangle & =\left(1+\alpha^{2}\right) \epsilon P_{0}^{2} / \beta,
\end{aligned}
$$

where $P_{0}$ is the reference momentum and

$$
\epsilon=\langle J\rangle
$$

is the transverse geometric emittance of the beam; the brackets $\langle\cdot\rangle$ denote an average over all particles in the beam. If the action of the particles is conserved along the beam line, then the transverse emittance defined by (11) will be conserved.

It is clear from the Hamiltonian in (7) that the betatron phase advance of the particle over the conditioning system depends on its position with respect to the reference particle. This is a benign effect from the point of view of beam conditioning, but it implies that the beta function varies with longitudinal coordinate, since

$$
\Delta \phi=\int \frac{1}{\beta} d s .
$$

In practice, the beta function has an energy dependence, which is related to the chromaticity of the beam line. In a conditioner, the "required" dependence of phase advance on longitudinal coordinate may be associated with an energy dependence, by introducing a correlated energy spread along the bunch, as we shall see in Sec. IVA. Thus, the energy dependence of the beta function may be used to give us the conditioning we require. However, there is an important point here: If the energy of the particles is changed at a location where the beta function has strong energy dependence, then the beam will become mismatched to the phase space, and an increase in the effective emittance will result. These apparently conflicting requirements (for the beta function to have some variation to allow the conditioning, and to be fixed to avoid emittance growth) may be resolved by zeroing out the variation of beta function with energy at those critical sections of the conditioner where the energy of the particles is changing.

The authors of Ref. [2] reached an incorrect conclusion that an effective increase in emittance is an inevitable consequence of conditioning. Their conclusion was based on an analysis of a conditioning Hamiltonian of a specific form that did not preserve the action as a constant of motion. If an alternative Hamiltonian, for example (7), is used, conditioning is achieved without an accompanying increase in emittance. In the case of the solenoid channel used in Ref. [2], the emittance was not conserved because a beam with large beta function was injected into a large solenoid field, creating large swings in beta function that did not cancel out for off-momentum particles. The lattice considered had too few free parameters to remove all of the energy dependencies at the critical locations.

\section{VARIOUS CONDITIONERS}

In this section we consider four possible realizations of conditioners that both preserve beam emittance and provide matching into the FEL along the length of the bunch.

\section{A. "Chromatic conditioner"}

Particle motion in a simple focusing channel may be approximately described by a Hamiltonian:

$$
H=\frac{\mu}{L} J+2 \pi \delta \frac{\xi}{L} J
$$

Here, $\mu$ is the phase advance over the length of the beam line for a particle with the nominal energy, and $\xi$ is the chromaticity defined by 


$$
\xi=\frac{1}{2 \pi} \frac{\partial}{\partial \delta} \Delta \phi
$$

where $\Delta \phi$ is the change in angle variable of a particle over the length of the beam line. We note that the Hamiltonian (13) is essentially the same as (7), which we used in our discussion of conditioning, but with the longitudinal coordinate $z$ replaced by the energy error $\delta$. This suggests we can construct a conditioner by first using an rf cavity to "chirp" the beam (i.e., introduce a correlation between longitudinal position and energy), then passing the beam through a focusing channel with some chromaticity, and finally using a second rf cavity to remove the chirp. The first of our conditions for a practical conditioner is satisfied by the extent to which the Hamiltonian (13) is a good approximation for the dynamics in the beam line. The second condition is satisfied if we are able to design the beam line in such a way as to make the Twiss parameters at the rf cavities independent of the particle energy. Note that it is not possible to satisfy the second condition everywhere along the beam line (since the chromaticity is related to the energy dependence of the beta function), nor is this necessary, as we shall demonstrate.

Physically, our conditioner will work as follows. The first rf cavity effectively makes the energy deviation of particles in the bunch a linear function of the longitudinal coordinate. In the beam line, particles "slip back" with respect to the reference particle by a distance depending on the betatron amplitude. This is a necessary effect of the chromaticity, as is easily seen from the Hamiltonian (13). The second rf cavity "corrects" the energy deviation introduced by the first cavity. However, the energy correction will not be that required to exactly cancel the energy deviation introduced by the first cavity, and there will be a residual energy deviation depending on the particle's betatron amplitude and the chromaticity of the beam line. This is exactly the conditioning that is required. Considering the effective emittance, if the beta functions at the rf cavities are dependent on energy, different "slices" of the bunch will become mismatched in terms of the transverse phase space distribution. Tuning the lattice so that the beta functions are independent of energy at the cavity ensures that the bunch remains matched along its entire length going into the FEL. The strength of the correlation is proportional to the slope of the rf gradient, which remains very nearly constant along the bunch for typical bunch lengths of the order of $100 \mu \mathrm{m}$.

As an illustration of this simple conditioner, we consider the beam line shown in Fig. 7. This uses thin quadrupoles alternately focusing and defocusing as in a FODO lattice, but the quadrupoles are tuned to minimize the variation of the Twiss parameters with energy deviation at either end of the beam line, where the (thin) rf cavities are placed. In calculating the beta functions, the beam line is assumed to be periodic. In principle, the amount of beam conditioning can be increased by repeating the beam line a number of times between the two rf cavities. Figure 7 shows the beta functions in both a "matched" case, in which the quadrupole strengths are adjusted to set the energy dependence of the Twiss parameters to zero at each end of the beam line, and in a "mismatched" case, where the Twiss parameters have significant energy dependence at each end of the beam line. Figure 7 also shows the energy dependence of the beta functions; this dependence is best given in terms of the $W$ function, which is defined by

$$
W=\sqrt{a^{2}+b^{2}},
$$

where

$$
a=\frac{1}{\beta} \frac{\partial \beta}{\partial \delta}, \quad b=\frac{\partial \alpha}{\partial \delta}-\frac{\alpha}{\beta} \frac{\partial \beta}{\partial \delta} .
$$

To understand the significance of the $W$ function, note that at a symmetry point in a beam line (as at either end of the focusing channel shown in Fig. 7) the symmetry condition enforces $\alpha=0$ at all energies, and we have then simply

$$
\frac{\Delta \beta}{\beta} \simeq W \delta .
$$

In other words, the fractional change in the beta function is just $W$ times the energy deviation.

In the matched case, the values of the $W$ functions at the start and end of the beam line are essentially zero. In between, the nonzero values indicate variation in the beta functions (and hence the phase advance) with energy, which is a necessary feature for the conditioning. In fact, the larger the values of the $W$ functions, the more conditioning is provided. Note that the required tuning of the quadrupoles may be achieved easily in a lattice design code such as MAD [10].

A conditioning beam line can be achieved by combining repeated cells of such a lattice, either in a chain or by insertion into a ring, with rf cavities at either end to apply and cancel an energy chirp. In Fig. 7 we show Twiss parameters for a properly matched case as well as a mismatched case with $\partial \beta / \partial \delta=30 \beta$ for the periodic beta function at the end points. The results of tracking simulations for the mismatched case after ten cells are shown in Fig. 8 for particles at longitudinal positions $z=$ 0 , $\pm 2 \mathrm{~mm}$. The mismatched case shows a strong dependence on $z$, caused by sensitivity to the energy chirp imparted by the first rf cavity. We note that the shapes of the ellipses in the horizontal phase space have been changed as a result of passing through the conditioner; this is consistent with the change in the Twiss parameters with the different energies of the different groups of particles. 


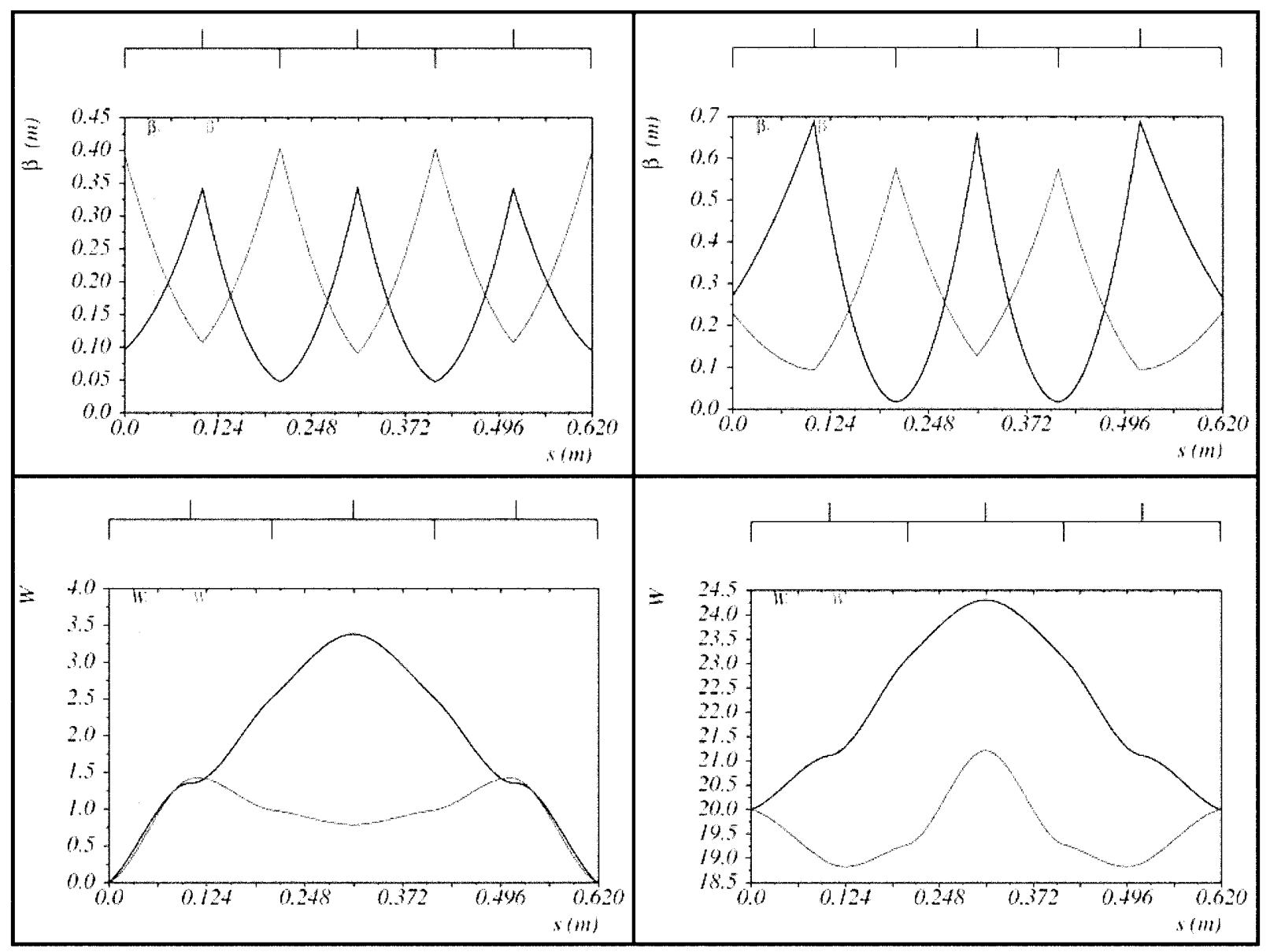

FIG. 7. Lattice parameters for a simple chromatic, conditioning beam line. The left-hand plots show the "matched" case, and the right-hand plots the "mismatched" case. Black lines correspond to the horizontal plane, red lines to the vertical plane. Top: Beta functions. Bottom: Variation of the above beta functions with energy, expressed in terms of the $W$ function.

Unfortunately, the amount of conditioning that may be provided by this simple chromatic conditioner with realistic parameters is not great. The parameters of the matched example are given in Table II. Note that from

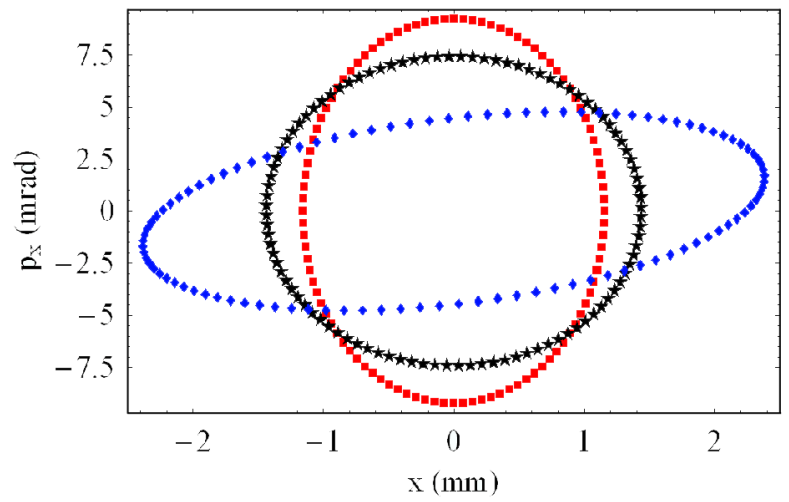

FIG. 8. (Color) Phase space ellipses in the horizontal plane after ten cells of the mismatched conditioning lattice, at three distinct longitudinal beam coordinates: $z=0$ (stars), $z=$ $2 \mathrm{~mm}$ (squares) and $z=-2 \mathrm{~mm}$ (diamonds). For the matched case, all curves coincide. the Hamiltonian (13) we find that the amount of conditioning in a chromatic conditioner is given by

$$
\frac{\Delta \gamma}{\gamma J}=-2 \pi \frac{e V_{\mathrm{rf}}}{E} \frac{\omega_{\mathrm{rf}}}{c} \xi
$$

Using the parameters in Table II, we find that the change in energy deviation for a particle with action $5.34 \mu \mathrm{m}$ is about 0.01 , in good agreement with tracking results. The amount of conditioning provided by this beam line is

TABLE II. Parameters in the example matched chromatic conditioning beam line.

\begin{tabular}{lcc}
\hline \hline Beam energy & $E$ & $1 \mathrm{GeV}$ \\
Normalized emittance & $\langle\gamma J\rangle$ & $5.34 \mu \mathrm{m}$ \\
rf voltage & $V_{\mathrm{rf}}$ & $52.4 \mathrm{MV}$ \\
rf frequency & $\omega_{\mathrm{rf}}$ & $2 \pi \times 4.85 \mathrm{GHz}$ \\
Max. quad strength & $k_{1} l$ & $15.2 \mathrm{~m}^{-1}$ \\
Beam line length & $L$ & $37 \mathrm{~m}$ \\
Chromaticity & $\xi$ & -57.4 \\
Conditioning & $\kappa$ & $0.0019 \mu \mathrm{m}^{-1}$ \\
\hline \hline
\end{tabular}


$1.9 \times 10^{-3} \mu \mathrm{m}^{-1}$, or about 3000 times smaller than that required, for example, by LCLS.

Conditioning in both planes can be achieved either with two lattices or by coupling the planes. We note that the Hamiltonian in (13) averaged the phase advance over the length of the beam line, whereas in a finite beam line there will be some dependence on initial betatron phase. However, while the conditioning parameter grows linearly with distance, the variation with betatron phase will remain bounded, and so it is not a serious problem. If the lattice is not properly matched for the variations in beam energy driven by the rf cavities, on the other hand, the variation with longitudinal position will get much worse as the conditioning increases.

\section{B. Solenoid-based conditioner}

Instead of using quadrupoles to focus the beam as in the simple chromatic conditioner, we can use a solenoid as proposed in Ref. [2]. The importance of matching the beam to the solenoid was observed by Kim at the University of Chicago and Argonne National Laboratory [11]. The basic arrangement and principles of operation are the same as for the chromatic conditioner: a solenoid is "sandwiched" between two rf cavities, with the cavities tuned to apply an energy chirp at the entrance to the solenoid, and cancel the chirp at the exit. In general, a particle follows a helical path through the solenoid, with the radius of the path determined by the horizontal and vertical coordinates of the particle at the entrance of the solenoid. For simplicity, let us consider a solenoid of length $L$ that is tuned such that the linear transfer matrix in the two transverse planes is the identity, i.e.,

$$
|k| L=2 n \pi,
$$

where $n$ is an integer and

$$
k=\frac{B_{z}}{B \rho},
$$

here $B_{z}$ is the longitudinal solenoid field and $B \rho$ is the beam rigidity. To match the beam to the solenoid, we need to impose the condition

$$
\beta=\frac{1}{k} .
$$

In this case, it turns out that the chromatic variation in the beta function is zero, as well as the beta functions being constant along the length of the solenoid. The change in longitudinal coordinate from a particle passing through a single solenoid is given by

$$
\Delta z_{1}=-\frac{1}{2} k^{2} L \beta\left[J_{x} \cos ^{2}\left(\phi_{x}\right)+J_{y} \cos ^{2}\left(\phi_{y}\right)\right] .
$$

If we consider two solenoids separated by a $\pi / 2$ betatron phase advance in both planes, then the combined change in longitudinal coordinate is

$$
\Delta z=-\frac{1}{2} k^{2} L \beta\left(J_{x}+J_{y}\right) .
$$

Now adding an rf cavity to chirp the beam at the entrance to the first solenoid, and a second rf cavity to cancel the chirp (in the case of zero betatron amplitude) at the exit of the second cavity, we find the following expression for the amount of conditioning provided:

$$
\frac{\Delta \gamma}{\gamma J}=\frac{e V_{\mathrm{rf}}}{E} \frac{\omega_{\mathrm{rf}}}{c} \frac{B_{z} L}{4 B \rho}=\frac{e V_{\mathrm{rf}}}{E} \frac{\omega_{\mathrm{rf}}}{c} \frac{n \pi}{2},
$$

where $J=J_{x}+J_{y}$. Although it is possible, in principle, to increase the amount of conditioning by using a large beta function, if the beta function is not properly matched to the solenoid there can be a strong dependence of the Twiss parameters on the energy deviation, and a growth in the projected transverse emittance.

As an example, we consider the parameters given in Table III. Note that $B_{z} L / B \rho=2 \pi$ in this example. These parameters give a conditioning of roughly $1.6 \times$ $10^{-6} \mu \mathrm{m}^{-1}$, which is about 6 orders of magnitude smaller than the amount of conditioning needed for the examples given in Sec. II.

\section{C. $\mathbf{T M}_{210}$ cavity conditioner}

Sessler, Whittum, and Yu [1] have suggested using an rf cavity operating in the $\mathrm{TM}_{210}$ mode. Having realized that transverse phase space mismatch can occur along the length of a bunch with nonzero length, they considered only a very short bunch. However, this mismatch may be avoided using the techniques described above, to eliminate the (first-order) dependence of the beta function on energy and thus on the longitudinal coordinate. The amount of conditioning provided by a conditioner based on a $\mathrm{TM}_{210}$ cavity may be written as

$$
\frac{\Delta \gamma}{\gamma J}=\frac{1}{\gamma} \frac{e E_{0}}{m c^{2}} \frac{\omega_{\mathrm{rf}}}{c}\left(\beta_{\max }-\beta_{\min }\right),
$$

where $E_{0}$ specifies the peak longitudinal electric field in the cavity,

$$
E_{z}=\frac{1}{4}\left(\frac{j_{21}}{R}\right)^{2}\left(x^{2}-y^{2}\right) E_{0} \cos \left(\omega_{\mathrm{rf}} t\right),
$$

for cavity radius $R$ and frequency $\omega_{\mathrm{rf}}$. The constant $j_{21}$ is the first zero of the Bessel function $J_{2}$. As an example, we consider the parameters given in Table IV. These parameters give a conditioning of roughly $270 \times 10^{-6} \mu \mathrm{m}^{-1}$,

TABLE III. Parameters for estimate of conditioning provided by a solenoid conditioner.

\begin{tabular}{lcc}
\hline \hline Beam energy & $E$ & $1 \mathrm{GeV}$ \\
rf voltage & $V_{\mathrm{rf}}$ & $10 \mathrm{MV}$ \\
Cavity frequency & $\omega_{\mathrm{rf}}$ & $2 \pi \times 4.85 \mathrm{GHz}$ \\
Integrated solenoid field & $B_{z} L$ & $20.96 \mathrm{Tm}$ \\
\hline \hline
\end{tabular}


TABLE IV. Parameters for estimate of conditioning provided by $\mathrm{TM}_{210}$ cavity conditioner.

\begin{tabular}{lcc}
\hline \hline Beam energy & $E$ & $1 \mathrm{GeV}$ \\
Cavity field amplitude & $e E_{0} / m c^{2}$ & $200 \mathrm{~m}^{-1}$ \\
Peak rf voltage & & $3.2 \mathrm{MV}$ \\
rf power $(Q=1000)$ & & $20 \mathrm{MW}$ \\
Cavity frequency & $\omega_{\text {rf }}$ & $2 \pi \times 4.85 \mathrm{GHz}$ \\
Beta functions & $\beta_{\max }$ & $30 \mathrm{~m}$ \\
& $\beta_{\min }$ & $5 \mathrm{~m}$ \\
\hline \hline
\end{tabular}

which is again small compared to the amount of conditioning needed for the examples given in Sec. II.

\section{FEL SIMULATION RESULTS}

Although the lattice with a $\mathrm{TM}_{210}$ cavity produces weak conditioning, unwanted nonlinear effects are sufficiently small to consider implementing it in a ring with many turns to provide the necessary conditioning. With each pass, the change in energy deviation resulting from the cavity is

$$
\begin{aligned}
\Delta \delta= & \frac{e E_{0}}{2 m c \omega_{\mathrm{rf}} \gamma}\left(\frac{j_{21}}{R}\right)^{2} \cos \left(\omega_{\mathrm{rf}} t\right)\left[2 \beta_{\max } J_{x} \cos ^{2}\left(\phi_{x}\right)\right. \\
& \left.-2 \beta_{\min } J_{y} \cos ^{2}\left(\phi_{y}\right)\right] .
\end{aligned}
$$

Now suppose we make $N$ passes through the whole system, starting from a different betatron phase each time, so we can average over all values of $\phi$. The total change in energy deviation is

$$
\Delta \delta_{N}=N \frac{e E_{0}}{2 m c \omega_{\mathrm{rf}} \gamma}\left(\frac{j_{21}}{R}\right)^{2} \cos \left(\omega_{\mathrm{rf}} t\right)\left[\beta_{\max } J_{x}-\beta_{\min } J_{y}\right]
$$

and after exchanging the $x$ and $y$ planes, and a second round of $N$ turns, the net energy shift is proportional to $J=J_{x}+J_{y}$, yielding

$$
N\left\langle\frac{\Delta \gamma}{\gamma J}\right\rangle=\frac{N}{2 \gamma} \frac{e E_{0}}{m c^{2}} \frac{\omega_{\mathrm{rf}}}{c} \cos \left(\omega_{\mathrm{rf}} t\right)\left[\beta_{\max }-\beta_{\min }\right] .
$$

This is written in terms of an "average conditioning per pass," noting that conditioning is only properly obtained in this system by making repeated passes through the system, including coupling between the $x$ and $y$ planes. The conditioning is limited by the strength of the fields in the rf cavity and the number of passes which can be taken.

As an example, we consider the parameters given in Table IV. These parameters give a conditioning per pass of roughly $270 \times 10^{-6} \mu \mathrm{m}^{-1}$, which is larger than that provided by the other methods described here, but is again small compared to the amount of conditioning needed for the examples given in Sec. II. Many thousands of passes would be required to provide the beam conditioning required for LCLS, for example. It may be possible to use a conditioning section as an insert in a small

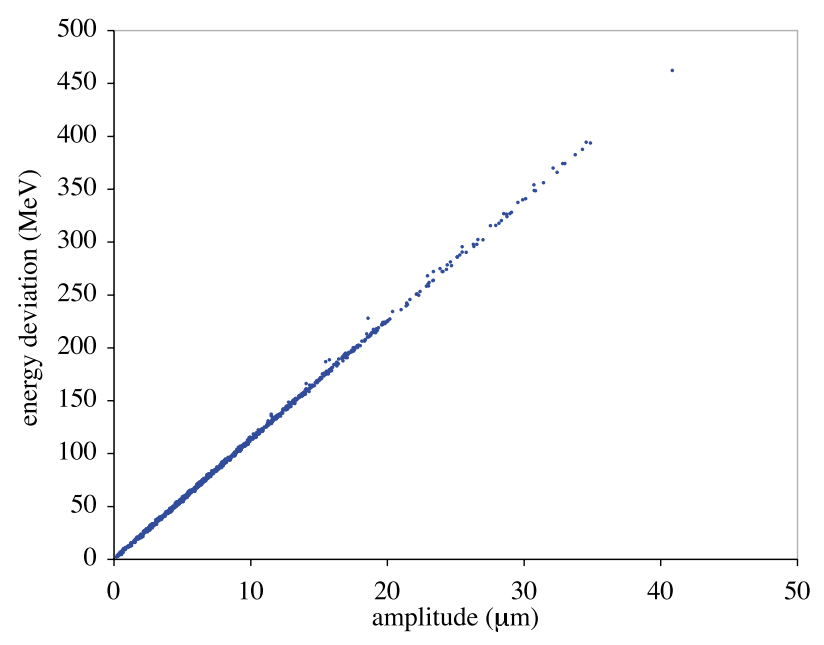

FIG. 9. (Color) Results of simulation of $\mathrm{TM}_{210}$ cavity conditioner. Correlation between energy and horizontal action after conditioning. The emittance growth is negligible.

storage ring; however, there are then a wide variety of effects that could disrupt the beam quality, including transverse and longitudinal nonlinear effects, and collective effects such as wakefields and coherent synchrotron radiation. The possibility of developing a practical conditioning ring requires further study.

To examine the question as to whether or not this lattice can produce a high-quality, conditioned beam, we simulate a ring incorporating the $\mathrm{TM}_{210}$ cavity conditioner. The particle tracking includes nonlinear terms in the magnetic fields and in the rf cavities, although the arcs are assumed not to induce any distortions in the beam (linear chromaticity is included). In addition to the

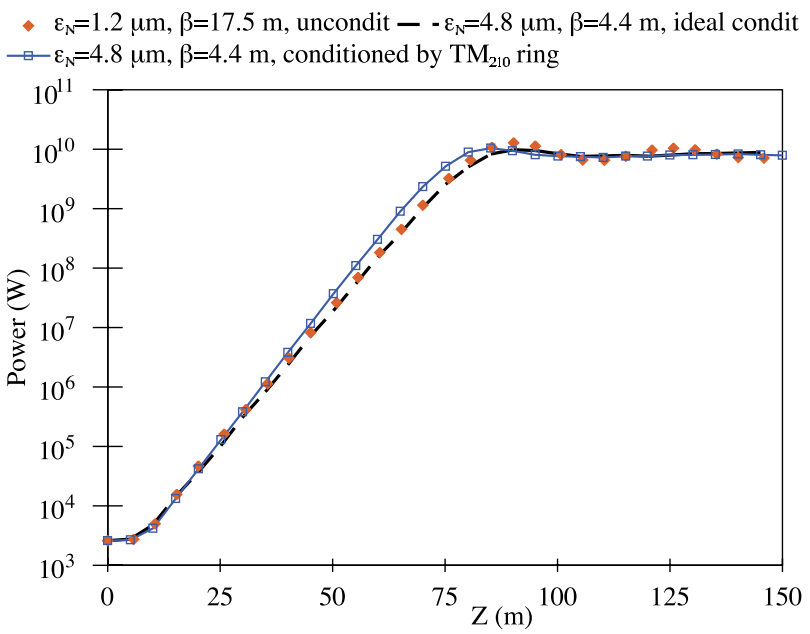

FIG. 10. (Color) Results of GENESIS simulations using LCLS parameters. FEL performance of $4.8 \mu \mathrm{m}$ emittance $(4 \times$ nominal) conditioned beam from $\mathrm{TM}_{210}$ cavity lattice (blue line with squares) is compared with performance of a similar, ideally conditioned beam (black dashed line) and of the nominal, unconditioned case (red diamonds); see Fig. 4. 
parameters listed in Table IV, the beam size is taken to have $\sigma_{z}=250 \mu \mathrm{m}$, peak current $150 \mathrm{~A}$, and relative energy spread $1 \times 10^{-4}$ at $1 \mathrm{GeV}$; subsequent bunch compression by a factor of 10 yields higher peak current and conditioning parameter, but with a higher energy spread as well, although the relative energy spread is reduced by acceleration to $14 \mathrm{GeV}$. The results are shown in Fig. 9, where the energy deviation is shown as a function of transverse amplitude. The required conditioning is achieved after 8500 turns in each plane; in this case, coupling has been introduced within the ring so that 17000 turns in one ring yield conditioning in both planes. Simulation results in GENESIS using this beam are presented in Fig. 10; the performance is almost identical to the idealized case presented in Sec. II C.

\section{CONCLUSIONS}

We have shown that beam conditioning in free-electron lasers allows for operation at significantly larger emittances than are envisioned for soft $\mathrm{x}$-ray FELs, the LCLS, and the so-called Greenfield $\mathrm{x}$-ray FEL sources. Previously perceived limitations to conditioning, based on an assumption that conditioning introduces a mismatch in the transverse phase space dependent on longitudinal position in the bunch, are readily avoided by a proper design of the conditioner. We demonstrate that the proper design of a conditioner requires that (a) the conditioning correlate energy with transverse action and that (b) the beam line Twiss parameters be independent of energy at points of the conditioner where the particle energy is changed. Under these conditions there is no increase in the effective emittance. We have three realizations of conditioning lattices using conventional technology, based on solenoids, quadrupoles, and specialized rf cavities. Conditioning at low energy is easier, but requires that the conditioning be preserved throughout the subsequent acceleration and transport. Conditioning at high energy requires a long conditioner section, leading us to propose a conditioning ring.

Finally, there is much work to be done. Conditioning rings need to be further explored, including radiation and collective effects; Greenfield FELs may benefit from an acceleration scheme that has conditioning sections built into it so that the beam emerges already conditioned. However, we have shown that by focusing on conservation of action and on variation of Twiss parameters with energy, the problem of developing a conditioning beam line can be reduced to more standard lattice design methods.

\section{ACKNOWLEDGMENTS}

We thank P. Emma, E. Esarey, W. Fawley, K.-J. Kim, and G. Stupakov for useful discussions. One possible laser-based method has been independently arrived at, and evaluated, by C. Schroeder, E. Esarey, and W. Leemans [3]. This work was supported by the Director, Office of Science, of the U.S. Department of Energy under Contract No. DE-AC03-76SF00098.

[1] A. M. Sessler, D. H. Whittum, and L.-H. Yu, Phys. Rev. Lett. 68, 309 (1992).

[2] P. Emma and G. Stupakov, Phys. Rev. ST Accel. Beams 6, 030701 (2002).

[3] C. B. Schroeder, E. Esarey, and W. P. Leemans, "Electron Beam Conditioning by Thomson Scattering" (to be published).

[4] R. Bonifacio, C. Pellegrini, and L. M. Narducci, Opt. Commun. 50, 373 (1984)

[5] S. Reiche, Nucl. Instrum. Methods Phys. Res., Sect. A 429, 243 (1999).

[6] R. Carr et al., in Proceedings of the 20th International FEL Conference (FEL98), Williamsburg, VA, 1998 (Elsevier, New York, 1998), Pt. II, pp. II-79-II-80.

[7] W. A. Barry et al., LBNL Report No. LBNL-51766 (2002).

[8] LCLS Design Study Group, SLAC Report No. SLAC-R521 (1998).

[9] J. Galayda, K.-J. Kim, and J. Murphy (unpublished).

[10] H. Grote and F. C. Iselin, CERN Report No. CERN-SLAP-90-13 (1990).

[11] K.-J. Kim (private communication). 